\title{
Medical Records
}

'By computerising health records, we can avoid dangerous medical mistakes, reduce costs and improve care'-George W. Bush, former US President (1946-)

\subsection{What Are Medical Records?}

The term of Medical Records is vast and vaguely defined. It encompasses, but is not necessarily limited to, the admission sheet, history sheet, progress of a patient as well as the charts of his or her vital parameters, intake-output data, medications given, referrals, and discharge summary. Medical certificates, birth certificates, Medico-legal case sheets also fall into this purview.

There is a lack of uniformity in the dimensions defined and encompassed by medical records in the literature.

\subsection{Is There a Legal Basis for Medical Records?}

In the USA, Medical Records refer to all communications related to a patient's physical or mental state that is recorded in any form and are maintained for diagnosis or treatment. These include medical records that are prepared by providers other than health professionals as well. However, they exclude material that is prepared in connection with utilization review, peer review, or quality assurance activities, irrespective of whom they are prepared by. They also do not include recorded telephone as well as radio calls to and from a publicly operated emergency dispatch office relating to requests for emergency services or reports of suspected criminal activity. The exceptions to this are communications that are recorded in any form or medium between emergency medical personnel and medical personnel regarding diagnosis or treatment. These inclusions and exclusions are pursuant to particular sections of their legal system. 
Analogous to that reference, medical records are acceptable in a court of law in India as per Sect. 3 of the Indian Evidence Act, 1872 amended in 1961. Amendment of the Consumer Protection Act (1986) in 1993 brought doctors under its purview following the landmark decision of the Honourable Supreme Court. Even its current version (most recently amended in 2019) does not specifically state what is included or excluded in Medical records. However, the onus of proving dereliction of service is on the consumer stressing the paramountcy of accurate, complete, and detailed records.

\subsection{What Are the Types of Medical Records?}

The WHO Medical Records manual enlists four major sections of medical records that include (Fig. 45.1):

- Administrative: Demographic and socioeconomic data.

- Legal: Such as consent.

- Financial.

- Clinical: Whether Inpatient/Outpatient; Elective/Emergency with relevant medical details.

Inpatient records can be in the form of:

- A History sheet/Emergency room admission record: which contains the patient's presenting complaints, duration along with details of symptoms, description of

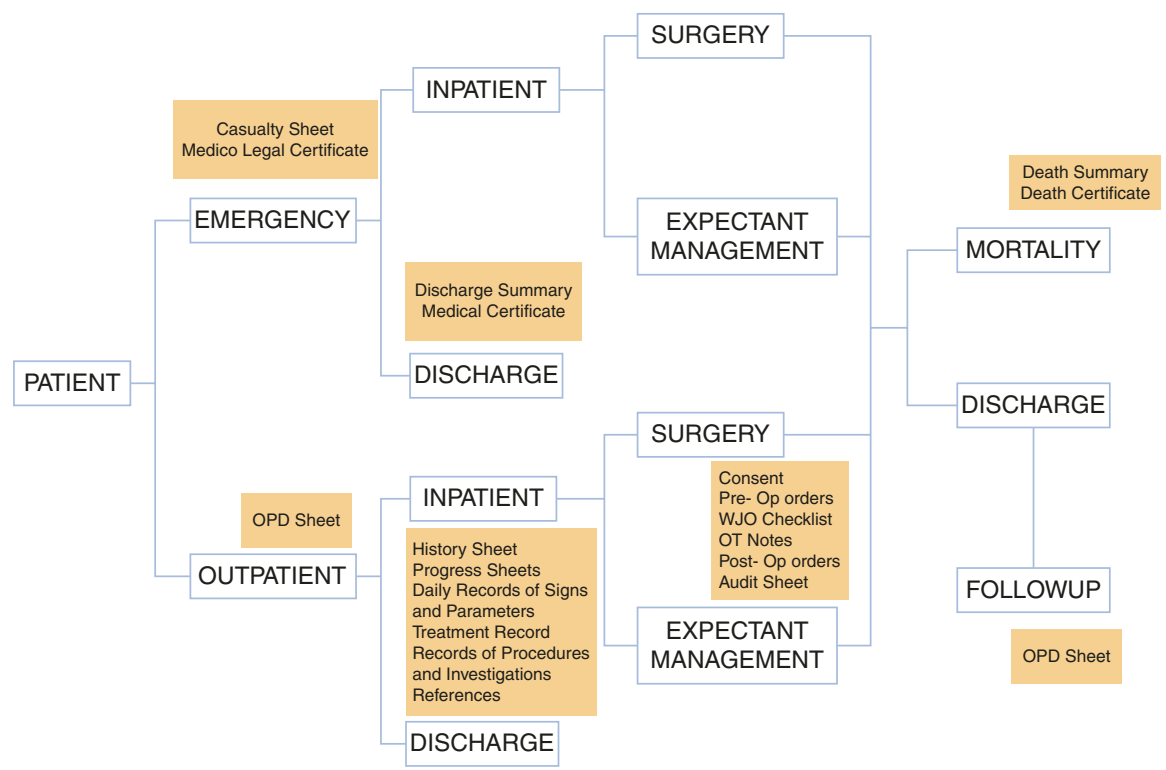

Fig. 45.1 Classification of inpatient and outpatient records 
signs, a provisional diagnosis, a record of investigations already done as well as those ordered along with a plan of treatment.

- Progress Sheets: These contain relevant information on the inpatient's clinical course in the hospital as well as parameters monitored and interventions carried out. Our routine clinical practice is recording it in the 'SOAP' format.

- Subjective: Symptoms.

- Objective: Signs.

- Assessment: Overall.

- Plan: of further investigations and treatment.

Besides the patient's complaints, the progress sheet must mention the postoperative day and procedure for patients that have undergone surgery.

- Operative and Post-operative notes: Essential components of the record of surgical patients are the detailed operative findings and steps of the procedure. It is useful to have a structured template that is followed in every patient which includes preoperative diagnosis, indications for operation, findings, procedure (in numbered consecutive steps), and post-operative instructions.

- Referrals: These form an important go-between for comprehensive patient management. Coordinated care with clear and crisp communications chronologically documented with relevant information provided and subsequent advice is crucial.

Each of these documents must be duly titled with the Patient's Name, Date, Bed, Ward, and who the case is seen by. They have no standing unless duly signed with a legibly written name and designation underneath.

- Discharge Summary: Arguably this is the single most important document of the health/illness a patient possesses, a status earned for its succinct comprehensiveness. As the name suggests it collates details of the course in the hospital in an abbreviated format ensuring meticulous documentation of the relevant information.

Formulation of minimum data sheets of each subdivision with features that reflect the identity of the assessee and assessor, time of assessment, enable the risk assessment of morbidity, and mortality with an objective score, are not only easy to record manually as well as electronically, but also improve clinical care.

\subsection{Are There Standards for Compliance for Medical Records?}

The National Center for Quality Assurance (NCQA), a not-for-profit organization in the USA that accredits health plans has developed a Healthcare Effectiveness Data and Information Set. It gives guidelines for medical record keeping. These consist 
of 21 points of which 6 are considered core elements (comorbidities, allergy, diagnosis, treatment, past history, and risk from procedures).

The National Accreditation Board for Hospitals (NABH) in India, provides standards for Information Management in the hospitals it accredits. Management of hospital information systems, as well as all modalities of information communicated to staff, patients, visitors, and the community in general, are included. Among the 7 standards it specifies in the fifth edition published in April 2020, it stresses on processes, accuracy, continuity of care, security of data, and information as well as their retention and retrievability. There is also a required review of medical records.

\subsection{What Is the Clinical Importance of Medical Records?}

Medical records are a repository of objective documented experience that can be extrapolated as tools for teaching, a basis for billing, and links to maintain continuity of care during follow up whether in the same or different institution by the same or a different practitioner. They are crucial to enable proper healthcare service delivery. Well-managed medical records allow smooth functioning and reduce the chances of human error. They are indispensable for audit and essential for research. In developing countries, where the majority of patients are poor and a significant proportion illiterate, they provide unambiguous insight into events that have transpired in previous encounters.

An Italian study by Poscia et al. [1] that aimed to investigate the correlation between the level of implementation of clinical governance dimensions and the quality of medical records along with the extent to which it could promote quality improvement in the context of a large Teaching Hospital found clinical governance influences health care quality, even in terms of the quality of medical records. It reiterates that well-kept medical records may be considered a surrogate marker of good clinical governance.

\subsection{What Is the Administrative Importance of Medical Records?}

The use of medical records surpasses current clinical practice and gains even more value in posterity. They are indispensable to medico-legal and malpractice suits where they have equal standing in defence and incrimination of health care providers and institutes. It is the legal liability of health care institutions to keep and maintain in standardized format indoor patient medical records for at least 3 years in India. In the USA, it is 6 years as per HIPPA (the Health Insurance Portability and Accountability Act), The Canadian Medical Protective Association recommends 10 years though it varies from jurisdiction to jurisdiction; Similarly, England requires retention up to 10 years after death and electronic records must not be destroyed. 
Medical records are often perceived as the reflection of the standard of care provided by the health care institute.

Ethical and meticulously detailed record-keeping form the cornerstone of resilient health systems. They even provide the epidemiological data on which national health policy as well as health expenditure may be based.

\subsection{How Do Medical Records Facilitate Research and Audit?}

Record keeping is integral to both Research and Audit. For research, whether retrospective or prospective, meticulous record of patient's demographic and clinical data is imperative. For meaningful retrospective research, only meticulously maintained clinical records may yield patterns that can help improve practice. For instance, to evaluate intra-operative parameters that may significantly contribute to leaks in pancreatico-jejunal anastomoses in a centre correlation can only be demonstrated if data such as the texture of the pancreas, size of its duct, size, and location of the lesion, vascular anatomy, anastomotic technique, size and type of suture used etc. are diligently documented. Similarly, the first step in Audit is reviewing one's own practice which is almost entirely hinged ongoing through past records. Also, to complete the audit cycle with re-audit, any demonstrable change is contingent on that change reflecting in records. Reviewing mortality files in regular mortality meets can help identify preventable deaths. Similarly, regular audits can help identify near misses. All of these together contribute to evidence-based and improved clinical care.

Digitalization of medical records has made Audit and Research significantly less cumbersome. Patient data is available a click away. Furthermore, maintaining data on spreadsheets allows one to use filters to specify research parameters. Also, if considered at the onset and planned, they even improve data portability.

Most national and international standards for maintaining and accrediting EMRs include a mandatory review of records.

\subsection{How Are Medical Records Maintained?}

Medical Records may be recorded both in an electronic or paper format. Most health care organizations tend to use a combination of both.

Paper records are traditional and widely kept. The responsibility and accountability are clearly allocated with the roles of data entry operators, doctors, and staff nurses defined.

The single largest value of electronic medical records (EMR) is in promoting transparency, followed by, perhaps immeasurable but definitely welcome, impact of legibility.

EMR directly improves retrievability and follow-up of patients.

Indirectly electronic records promote improved clinical governance, mitigating not only pilferage and fabrication but also human error. 
Electronic medical records can be stored in various forms including a simply scanned copy of paper documents. Most hospitals utilizing electronic medical records have a specially designed software (Hospital Information System; HIS). In a study affiliated with the Australian Institute of Health Information conducted in four Saudi hospitals [2] collected information directly from different categories of health care professionals. It enumerated, validated, and ranked 10 benefits of an HIS-(1) Improved information access, (2) Increased health care professionals' productivity, (3) Improved efficiency and accuracy of coding and billing, (4) Improved quality of health care, (5) Improved clinical management (diagnosis and treatment), (6) Reduced expenses associated with paper medical records, (7) Reduced medical errors, (8) Improved patient safety, (9) Improved patient outcomes, and (10) Improved patient satisfaction.

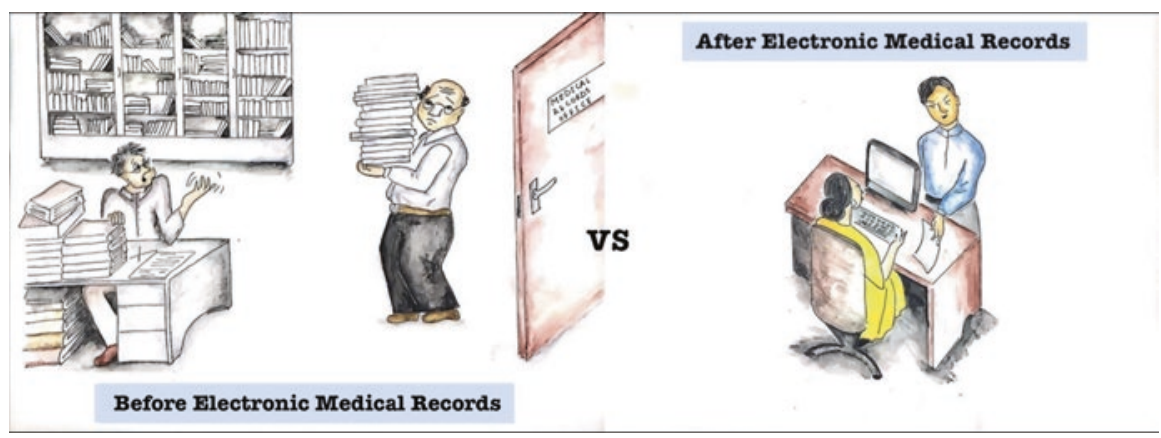

\subsection{How Are Electronic Medical Records (EMR) Different from Paper Records?}

When comparing Electronic records with paper records, electronic ones score in ease of retrieval, perusal, maintaining continuity, uniformity as well as storage. They also allow performance analysis besides audit.

When its advantages are extrapolated beyond logistics, EMR simplifies and increases the feasibility of research with keyword searches, data at one's fingertips which can be easily transferred, sorted, and processed. These also improve legal standing with digital footprints that are time and date stamped and verifiable.

The switch from paper to digital can be slow, inconvenient, and nagging; the initial transition is marred by a lack of familiarity with both hardware and software, but the period is brief and worth the initial investment in training, hardware, software, and personnel. The benefits become self-evident in a very short time.

Paper records retain their significance in feasibility of recording even in remote areas, familiarity, and freedom from risk to intangible threats like technical glitches, viruses, and hacking. However, their universality is challenged by the concept of 'cloud' databases that allow information to be accessed from anytime anywhere in real time. 


\subsection{Why Is Medical Record-Keeping Important in Developing Countries?}

A wealth of data already exists in the developing world, a direct corollary of the heavy burden of disease and difficult access to healthcare as well as a paucity of resources - health related and otherwise. The challenge remains in leveraging systems so crutches can become strengths. One of the advantages of EMRs is to link direct patient care processes within the information systems in health care delivery with the information systems at the level of government initiatives and international programmes. This can expedite improvement in health indicators with real-time access to data and its monitoring and evaluation. Digitalization in health care is here to stay. EMRs are already widely adopted in the developed world and their benefits are self-evident. Databases developed, centralized or institutional, have not only enabled authentic research and publication but demographic and disease-related information can guide national health policies, enable institutions, states, and nations to develop risk assessment models that are specific and applicable to their regional population. The major technical challenges in the adoption of EMR in developing countries include poor system security as well as a lack of backups for power, data, and technical support.

\subsection{What Are the Challenges to Adoption of EMR in Developing Countries?}

The Global Observatory for eHealth (GOe) has been set up by the World Health Organisation with the intent to examine the implementation of eHealth in the Member States along with the benefits of adopting information and communication technologies to health care services. A survey by the GOe found most patient data are collected on paper in spite of the high costs, limited usefulness, and inefficiencies though worldwide, a lot of countries collect aggregate health data in electronic formats at the national level. Countries in the upper-middle-income and highincome (World Bank groups) have greater adoption of EMR than those in the lower groups. Only a limited number of countries report widespread use of electronic formats and communications. These mostly include high-income countries with advanced information and communication technology infrastructure along with a workforce most of which have received advanced education. In these countries, electronic transmission use is similar to electronic capture rates which could be indicative of the adoption of interoperable systems to communicate electronic data. There is widespread availability of mobile telephone communications technology. This is an important asset that can stimulate the deployment of electronic patient health information. Many countries have adopted standards for data interoperability and have national plans for implementation. Developing economies such as Brazil, China, and India are beginning to incorporate EMRs into their health systems. However many factors still hinder their widespread adoption in other economies. For example, in sub-Saharan Africa, cost of the procuring and maintaining EMR, inadequate power supply, and Internet access form major hurdles. 


\subsection{What Are the Technological Advances in Medical Record Keeping?}

The practice of telemedicine has seen an exponential rise and the guidelines of practice have been rolled out already. This is also coupled with the increasing proportion of surgical procedures carried out using minimal access technology and hence available video recordings. With a rise in the number of medico-legal cases, there is also a trend towards video recording the patient's consent as well as important briefings regarding surgery - the procedure, its complications as well as alternatives. Besides widely available cellular phones with cameras, a lot of records are available in the form of photos or videos. There are many systems in use now. EPIC, a privately owned healthcare software company, founded in Wisconsin, United States is quite popular in North America as well as the UK. There are also other, even cheaper models. In a study conducted in Boston [3], aimed at understanding how clinicians utilize image uploading tools in a home-grown EMR system, general surgery was amongst the departments uploading the most clinical images. The recommendations from that publication include training on when to consider adding photos to the record, how to take good clinical photos, and policies for uploading and protecting images. They also address the quandary regarding the protection of photos taken using a personal smartphone during transfer. Their hospital has developed a CliniCam, an iOS mobile application for taking clinical photographs and securely uploading those images onto the patients' medical records. Similar to this, there is an Indian application and web-based software 'Raxa' available for iOS as well as android that is being used by our department. It can store patient's demographic data, imaging investigations as well as daily patient progress. The information stored is accessible to health care providers as well as patients.

With the digitization of records, vast volumes of data are available and this is going to increase with the passage of time. The use of big data in health analytics, especially in the fields of improvement of the quality of care for a whole population, predicting new epidemics, and ensuring equal access to care for everyone has tremendous potential. A case study in Morocco [4] concluded while adopting electronic medical records, considering the adoption of a framework based on big data storage and processing technologies to attain high-performance potential descriptive, predictive, or prescriptive analytics can be incorporated easily to get valid significant insights from the EMR data.

\subsection{What Is the Future of Medical Record Keeping in South Asia?}

Most South Asian Countries have begun adopting Electronic Medical Records, however, the adoption is neither uniform nor universal. There is a centrally driven mandate in countries such as India, China, and Pakistan to not only digitalize medical records but also improve integration and connectivity. Despite its economic progress, most regions of China have EMRs developed in an uncoordinated manner. 
A case study [5] published in 2019 explored bottlenecks of data integration and recommended the adoption of a multi-stakeholder participation in data collection followed by establishment of standards for information and an audit mechanism. In Pakistan, a legislation governing the use of the national Electronic Health Record system came into being in 2013. In India, the Electronic Health Record standards were last updated in 2016 and Telemedicine practice guidelines rolled out in 2020; in part expedited by the COVID-19 Pandemic. All of this is in line with the National Digital Health Mission and NITI Aayog's proposal of 'National Health Stack' with the goal of developing digital health records for all citizens by 2022. The pandemic has also seen momentum build-up for improving adoption of Health Information System and EMR in Bangladesh. In a paper [6] out of Sri Lanka researching gaps in national EMR implementation pointed out the current EMR implementations in public sector (non-profit organizations) has provided significant cost benefits. It also pointed towards the limited scientific research on EMRs, issues related to data ownership, private and security, need for furthering data analytics and underscored the importance of national policy for successful implementation of countrywide digital system.

EMRs have been around for a while now, albeit in a fragmented manner. As identified, the questions of Data security and Privacy, even ownership still need to be addressed more rigorously in all of these countries. For example, in India, the Right to Privacy is recognized as a fundamental right and the guidelines are that health care practitioners protect the confidentiality of their patients under the IMC regulations, IT Act 2000, SPDI Rules 2011, and other data protection laws as applicable. The Electronic Health Record Standards 2016 are important guidelines that are relevant to data protection. However there are still lacunae and loopholes that may be exploited such as even though consent is necessary for a telemedicine consultation, there is no specification requiring consent for processing, storing, and transferring patient's medical records and health information.

\subsection{How Can One Maintain Records in a Department?}

Even though not encouraged specifically, maintaining patient files is integral to the clinical training of doctors (Fig. 45.2). A well-written history and a comprehensive physical examination are taken as a mark of competence in residents and regularly updated progress sheets, as a reflection of their responsible attitude towards patient care. Despite the central role it plays, the teaching in this sector is informal and more of an oral tradition than dedicated practice. With rising importance due to growing number of litigations as well as the advent of EMRs, record keeping is becoming increasingly demanding. Medical record keeping is growing as a branch of its own with increasing admissions and electronic record keeping, becoming more effort intensive. Where patient sheets continue to be maintained by clinicians, their storage, duplication, or scribing them into electronic systems is increasingly being taken over by data entry operators. Familiarity and a basic knowledge of medical terms, procedures, and legal requirements, as well as structure is important. 
MAINTAINING RECORDS IN A DEPARTMENT

Review current system Identify Gaps

Adopt a standard practice

Execute

- Materials

- Personnels

- Accountability
MAINTENANCE

APPLICATION

\section{Processes \\ Personnel \\ Materials}

Clinical Teaching

Audit

Research

Legal Review

Financial Efficiency

Public Health Policy

Fig. 45.2 Maintaining medical records: An overview

There are around 21 paramedical colleges in India offering Medical Record Technology courses. The number of medical colleges in India offering MBBS is 529 (National Health Profile 2019).

\subsubsection{Initiation}

Medical records are a byproduct of clinical care of the patient and communication between the doctor-patient as well as doctors with other doctors and doctors with paramedical staff about the patient.

The key role they play requires attitude revision to treat them as an integral part of clinical care.

The first step to improving surgical record keeping would be an audit of one's current practices, choosing a standard to comply/developing one's own minimum data sets and identifying gaps.

It is also important to identify materials and personnel recruited, their roles and responsibilities for execution as well as assigning accountability to enable compliance and re-audit to complete the cycle.

Materials:

- Clinical sheets and Charts.

- Discharge summaries.

- Operative Notes and Details.

- Histopathology Reports.

- Videos/Photographs. 
- Mortality and Morbidity Data.

- Audits/Annual Reports.

- Hospital Information System/Computer with software such as Word/Pages/ Excel/Numbers etc.

Personnel:

- Number.

- Designation.

- Roles and Responsibilities.

Accountability:

- Head: Oversight.

- Consultants: Regular checks/countersign.

- Residents: Clinical Details.

- Nurses: Emergency (Treatment and Vital charts, Admission/Discharge/Transfer statistics), Ward (Files, Monitoring and Treatment Charts) OT (Record of cases, specimens, special events);

- Paramedical Staff: Data entry/duplication/storage.

- Institute: Medical Records Department.

\subsubsection{Maintenance}

Once adopted, the aim is to inculcate it in daily routine practice so the process becomes continuous. Most important aspect to prevent fractures is to retrain as the department cycles with turnover of new personnel, especially residents. Datasets and processes pinned on the department notice board/duty room/ward are helpful. Accountability and review which may be in the form of regular audits also boost improved record keeping.

\subsubsection{Application}

The effort-intensive nature of meticulous record-keeping merits it contributes to patient care and improved clinical service as well be of benefit to health care professionals.

Maintaining detailed records of not only patient data, but also their laboratory investigations, imagining studies etc. can aid retrospective meaningful research as well as audit, especially into perceived errors or mortality and even help identify near misses. Increased adoption Health information systems and EMR has made this conveniently feasible as it facilitates linking across platforms (laboratory services, radiology, pharmacy etc.) 
Minimum data sets improve resident training, completeness of records facilitates research and audit coupled with former two facets improve clinical service benefiting the department as well as the patient.

\subsection{Conclusion}

Medical records are pivotal to clinical care delivery. Digitalization is the way forward and facilitates improved Clinical Teaching, Audit, Research, and Legal Review. Big data generated can also facilitate better financial efficiency and guide Public Health Policy.

\section{References}

1. Poscia A, Azzolini E, Cacciatore P, de Belvis AG, Volpe M, Cambieri A, Damiani G, Ricciardi W, Specchia ML. Does Clinical Governance influence the quality of medical records? Ann Ist Super Sanita. 2018 Apr-Jun;54(2):104-8. https://doi.org/10.4415/ANN_18_02_05.

2. Khalifa M. Perceived benefits of implementing and using hospital information systems and electronic medical records. Stud Health Technol Inform. 2017;238:165-8.

3. Ai AC, Maloney FL, Hickman TT, Wilcox AR, Ramelson H, Wright A. A picture is worth 1,000 words. The use of clinical images in electronic medical records. Appl Clin Inform. $2017 \mathrm{Jul}$ 12;8(3):710-718. https://doi.org/10.4338/ACI-2016-10-RA-0180. PMID: 28696480; PMCID: PMC6220686.

4. Khennou F, Khamlichi YI, Chaoui NEH. Improving the use of big data analytics within electronic health records: a case study based OpenEHR. Proc Comput Sci. 2018;127:60-8. https:// doi.org/10.1016/j.procs.2018.01.098.

5. Wang Z. Data integration of electronic medical record under administrative decentralization of medical insurance and healthcare in China: a case study. Isr J Health Policy Res. 2019 Apr 1;8(1):24. https://doi.org/10.1186/s13584-019-0293-9. PMID: 30929644; PMCID: PMC6442402.

6. Sarathchandra K, Rathnayake S. Implementation challenges and Research Gaps of Electronic Medical Records (EMR) in Public Sector Hospitals of Sri Lanka. Int J Sci Research Pub (IJSRP) 9(7) (ISSN: 2250-3153). https://doi.org/10.29322/IJSRP.9.07.2019.p9124

Open Access This chapter is licensed under the terms of the Creative Commons Attribution 4.0 International License (http://creativecommons.org/licenses/by/4.0/), which permits use, sharing, adaptation, distribution and reproduction in any medium or format, as long as you give appropriate credit to the original author(s) and the source, provide a link to the Creative Commons license and indicate if changes were made.

The images or other third party material in this chapter are included in the chapter's Creative Commons license, unless indicated otherwise in a credit line to the material. If material is not included in the chapter's Creative Commons license and your intended use is not permitted by statutory regulation or exceeds the permitted use, you will need to obtain permission directly from the copyright holder.

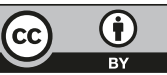

